\title{
Comparative Study between Primary Surgical Intervention and Conservative Management in Cases of Jones Fracture of Base $5^{\text {th }}$ Metatarsal Bone
}

H.A.Bassiooni, A.A.Ahmed, M.S.Abou Zied and H.A.Elgamal

Othopedic Surgery Dept., Faculty of Medicine, Benha Univ., Benha, Egypt

E-Mail:hossam@gmail.com

\begin{abstract}
The fifth metatarsal base fracture is the most common fracture of the forefoot. The aim of this study was to evaluate the short term results of comparison between surgical and conservative management of Jones fracture of base $5^{\text {th }}$ metatarsal: This is a comparative study included forty patients, twenty patients were treated by percutaneous fixation using cannulated $4.0 \mathrm{~mm}$ screw and twenty patients were treated conservatively with below knee cast. The duration from injury to admission to the hospital ranged from 1 to 3 days with an average of 2 days. The average follow-up duration was 6 months (range from 4 to 12 months). The American Orthopedic Foot and Ankle Society (AOFAS) used as a clinical rating system to monitor improvement following injury was done at 6 weeks, 12 weeks and 6 months. Patients treated with percutaneous screw fixation all achieved full union and had AOFAS score ranged from 91 to 98 at end of follow up at 12 weeks, while patients treated conservatively by cast had AOFAS score ranged from 73 to 87 at end of follow up at 12 weeks. After calculating $\mathrm{P}$ value to compare both groups according to end of follow up AOFAS Score $\mathrm{P}$ value $=0.000665781, \mathrm{P}$ value significant $<0.001$ which is highly significant. Early screw fixation is safe and effective method for treatment of acute Jones fracture of base of $5^{\text {th }}$ metatarsal.
\end{abstract}

Keywords: Jones fracture, $5^{\text {th }}$ metatarsal bone, Nylon Tape sutures-Ethibond suture.

\section{Introduction}

Fractures of the fifth metatarsal present a unique set of challenges for treatment to the foot and ankle specialist. Understanding the local anatomy, vascular supply, function, and dynamic stresses placed upon the bone, as well as fracture classifications, mechanisms of injury, and expected responses to treatment aid immensely in the decision-making processes.

Rammelt et al stated it to be the most common fracture of the forefoot[2]. Up to $70 \%$ of metatarsal fractures involve the fifth metatarsal and $80 \%$ of fifth metatarsal fractures involve the proximal aspect of the bone $[1,3]^{\text {T The }}$ Thactures have a greater incidence in males in their third decade and females in their seventh decade, with a greater prevalence in women with low bone mineral density[4]. Stress fracture of the fifth metatarsal bone is a common injury in athletes [5].

These injuries were initially described in 1902 by Sir Robert Jones in a series of six cases including one in his own foot [6].

The management of the fifth metatarsal's base fracture has been pursued by either operative, or non-operative measures. The operative measures usually include an internal fixation approach with an intramedullary, bicortical screw and/or bone graft-inlay. Whereas, the nonoperative measure employs an immobilization cast aimed to facilitate passive healing with/without weight-bearing. The choice of strategy to be utilized, is usually inclined upon the various classifications of fracture reported in the literature [7].

We tried to link theoretical information with our clinical study by comparing conservative and operative treatment in forty patients having fracture base of fifth metatarsal bone in variable ages, classification and mechanism of injury.

Clinical studies comparing conservative treatment versus surgical treatment are few. Therefore this clinical study was performed to evaluate this comparison.
The aim of this study was to evaluate the short term results of comparison between surgical and conservative management of Jones fracture of base $5^{\text {th }}$ metatarsal.

\section{Patient and method}

This is a comparative study included forty patients, twenty patients were treated by percutaneous fixation using cannulated $4.0 \mathrm{~mm}$ screw and twenty patients were treated conservatively with below knee cast.. The average follow-up duration was 6 months (range from 4 to 12 months). There were 23 males and 17 females. The age of the patients ranged from 18 to 60 years. All of the 40 patients had Jones fracture zone II of base of $5^{\text {th }}$ metatarsal. 13 patients were due to motor bike accidents and 27 patients were due to ankle twist. There were 24 patients right sided and 16 patients left sided.

\subsection{Inclusion criteria}

- Age group: 18 - 60 years.

- Fracture type: Jones fracture type II of base of 5th metatarsal.

- Gender: male and female.

- Closed fractures.

\subsection{Exclusion criteria}

- Type I and type III fracture base of 5th metatarsal.

- Age $<18>60$ years.

- Open fractures.

- Pathological fracture.

- Skeletal immaturity.

- Diabetic patients.

- Patients who were missed during follow up. 


\subsection{Cast group}

A below knee cast was applied and instructions that no weight bearing is allowed for the $1^{\text {st }}$ six to eight weeks. Cast was removed after 6 to 8 weeks and follow up X-rays were done to follow up union of the fracture and instructions to partial weight bearing as tolerated with crutches. X-rays were done at 2 weeks,4weeks ,6 weeks and12 weeks. AOFAS score was done at 6 weeks and 12 weeks to evaluate patient's pain and functional outcome.

\subsection{The operative group}

The patients were assessed for fitness for surgery by history, clinical examination and routine pre-operative laboratory investigations.

\section{Surgical technique}

\section{- Anesthesia}

All the patients were anaesthetized by spinal anesthesia.

\section{- Position}

Patients were operated on a standard radiolucent orthopedic table, in supine position under image intensifier guidance.

A stab incision about 0.5 to $1 \mathrm{~cm}$ proximal to the base of the fifth metatarsal bone. After the incision, a $4.0 \mathrm{~mm}$ cannulated screw guide pin was inserted into the space between the plantar fascia and the peroneus brevis tendon under image guidance.

After the guide pin was inserted, its position is checked under the image intensifier, we took several images as Anteroposterior, Lateral and oblique, to be sure the pin is in the intra medullary canal of the fifth metatarsal.

The patient remains in the hospital overnight, and prophylactic parenteral antibiotics are administered for the first 24 hours postoperatively.

Patients were instructed not to weight bear for first 2 weeks then instructed to partial weight bearing with crutches till 4 weeks. Then After 4 weeks, the patient starts on full weight-bearing without any aids. However, if we found any loosening over the screw or widening of the fracture gap, then we wait for another 2 weeks till full weight-bearing. After 12 weeks there were no restrictions for the patient for running or contact sports .

The American Orthopedic Foot and Ankle Society (AOFAS) [8] used as a clinical rating system to monitor improvement following injury was done at 6 weeks, 12 weeks and 6 months.

Data were statistically described in terms of mean standard deviation (SD), and range, or frequencies (number of cases) and percentiles when appropriate. Comparison of numerical variables between the different time periods was done using Freidman's test with multiple posthoc pairwise comparison tests. $p$ values less than 0.01 was considered statistically significant. All statistical calculations were done using computer program SPSS (Statistical Package for the Social Science; SPSS Inc., Chicago, IL, USA) release 15 for Microsoft Windows (2006).

\section{Results \\ 3.1The Cast group}

The sample size was 20 patients, 15 patients achieved full union, one patient was complicated by Sundeck's atrophy, 5 cases were considered treatment failure diagnosed as nonunion, two cases were operated upon by screw fixation and grafting, one was treated by mini-plate and grafting, the remaining two refused the operation.

Our patients' ages ranged from 18 to 60 years, we put each patient in its age group to compare results of each group in the same management.

The time of union was correlated with the time of return to normal activity, painless movement of the foot and radiological assessment for union, Patients achieved union ranging from ten to fifteen weeks with average twelve weeks.

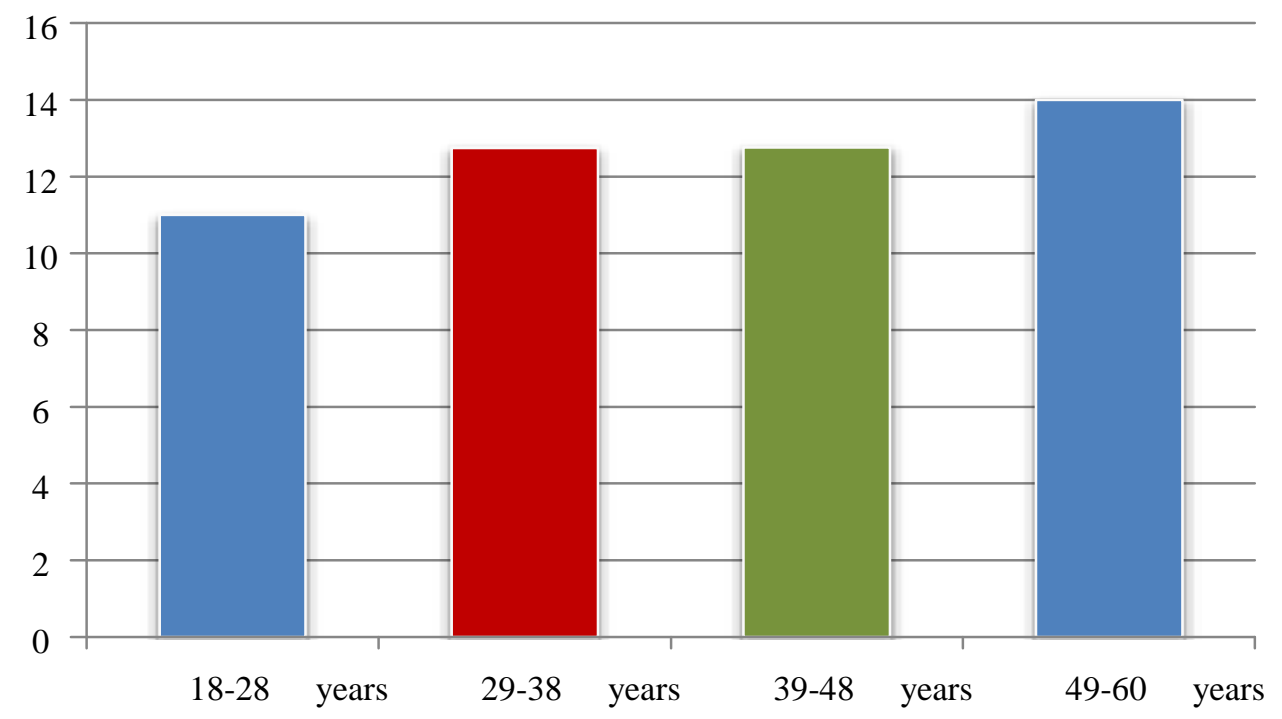

Fig (1) graph showing time of union in weeks in cast group. 


\subsection{The operative group:}

The sample size was 20 patients, all patients achieved full union, one patient was complicated by superficial infection treated by antibiotics.
The time of union was correlated with the time of return to normal activity, painless movement of the foot and radiological assessment,

Patients achieved union ranging from six to nine weeks, average seven weeks Fig (2).

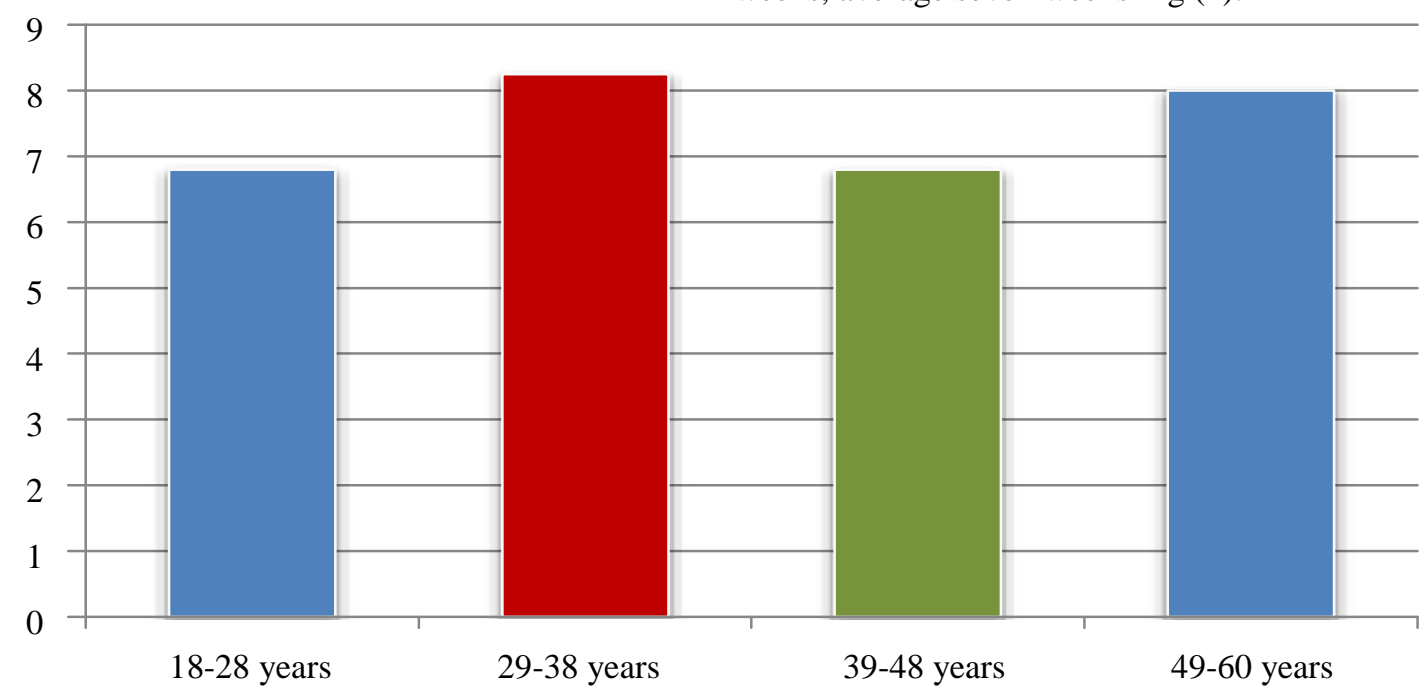

Fig (2) Graph showing time to union in weeks in operative management.

Patients treated with percutaneous screw fixation all achieved full union and had AOFAS score ranged from 91 to 98 at end of follow up at 12 weeks, while patients treated conservatively by cast had AOFAS score ranged from 73 to 87 at end of follow up at 12 weeks. After calculating $\mathrm{P}$ value to compare both groups according to end of follow up AOFAS Score $\mathrm{P}$ value $=0.000665781, \mathrm{P}$ value significant $<0.001$ which is highly significant.

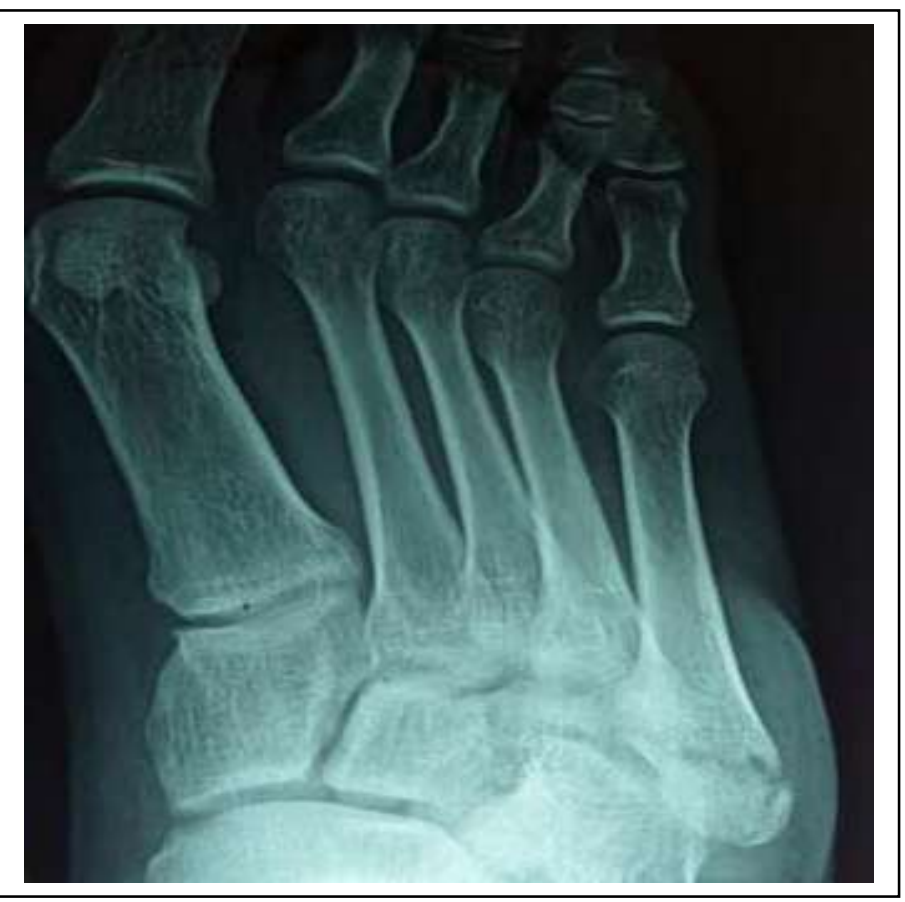

(A)

\section{Case presentation} Case (1)

25 years old male with fracture right fifth metatarsal in zone II, the mode of trauma was motor bike accident and was associated with sprain ankle. He was operated on $2^{\text {nd }}$ day.

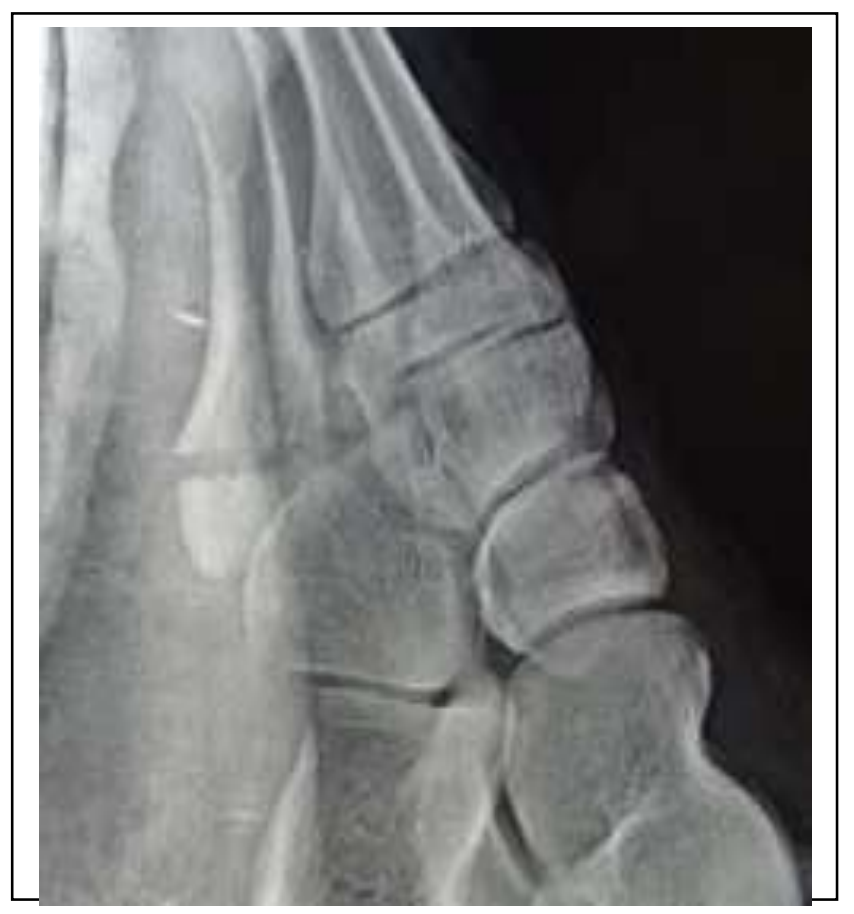

(B)

Fig (3) (A)Anteroposterior view of patient preoperatively in zone II.(B): Lateral view of the patient pre-operatively in zone II. 

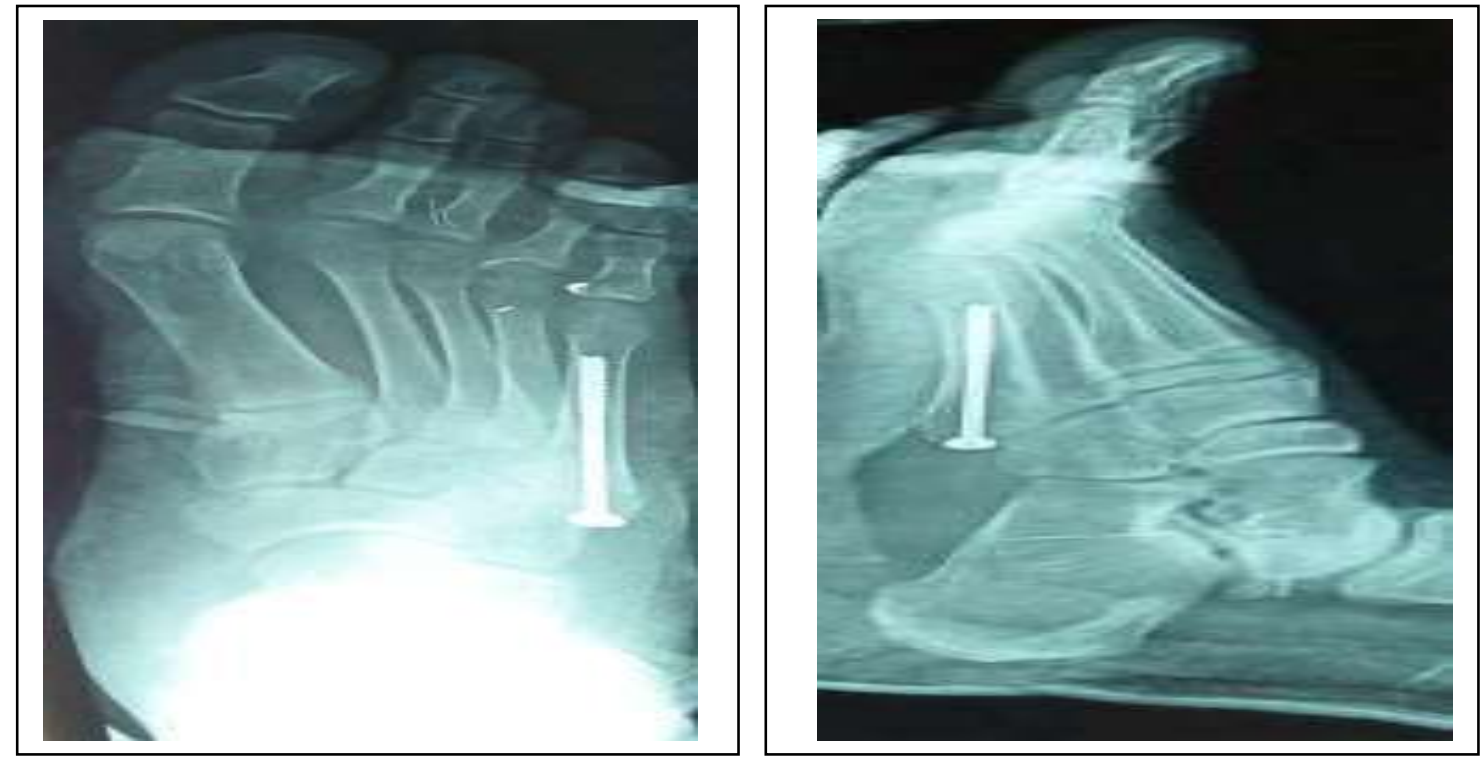

Fig (4) Post-operative Anteroposterior and Lateral views of the patient post-operatively in zone-II.

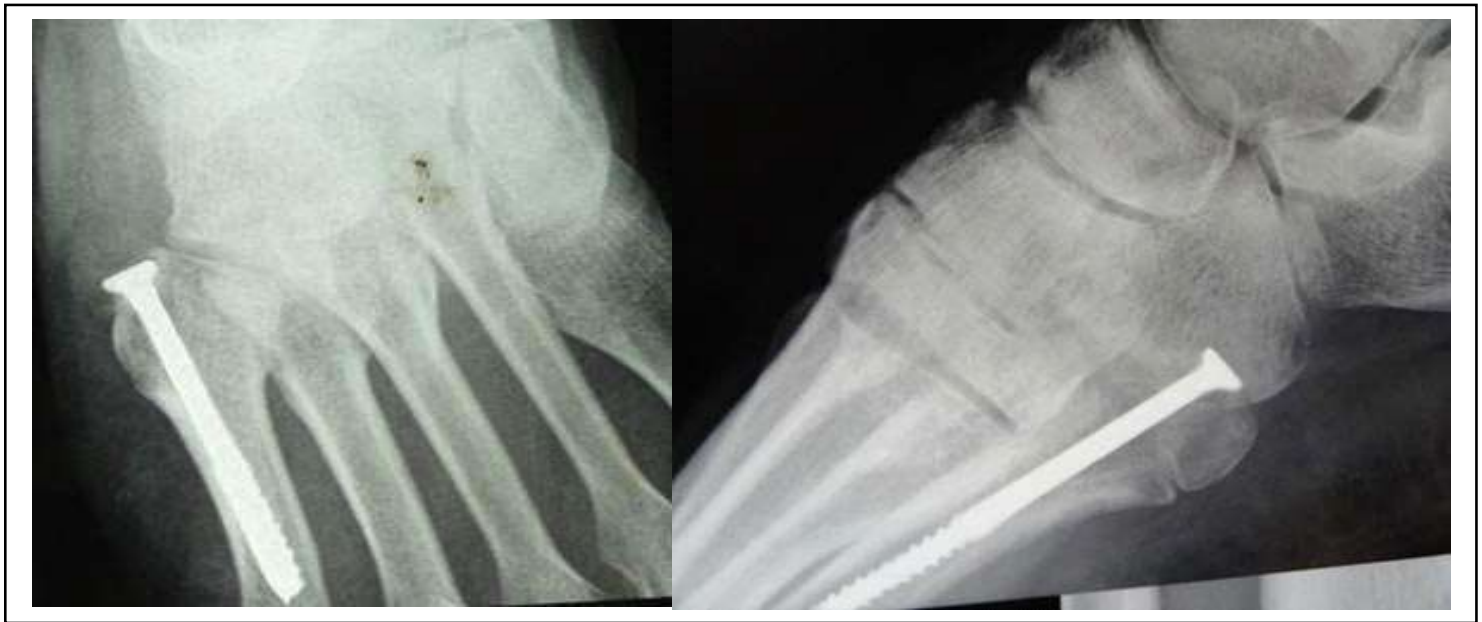

Fig (5) Anteroposterior and Lateral views of the patient after healing at 8 weeks.

Case 2

25 years old male patient with fracture right fifth metatarsal in zone II, the mode of trauma was ankle twist. She was treated conservatively in cast.

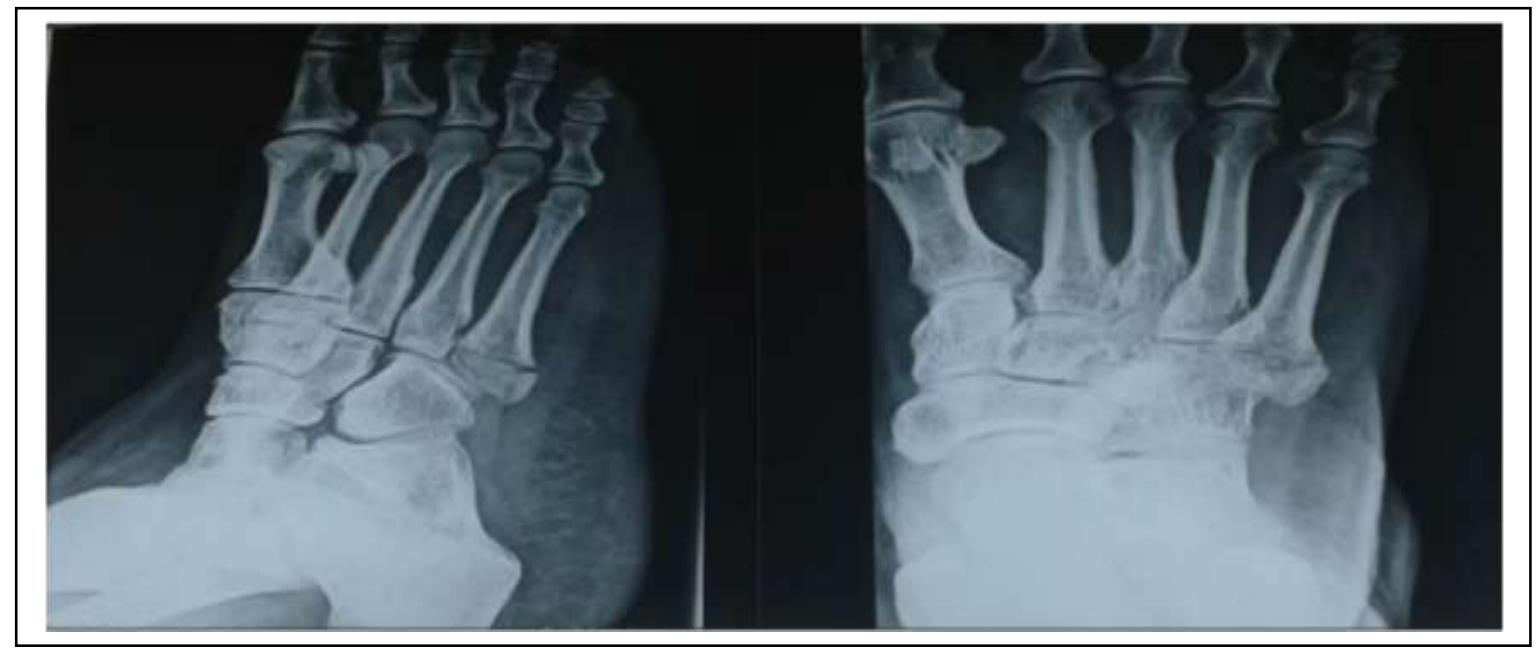

(A)

(B)

Fig (6) (a) Oblique and (b) Anteroposterior views showing fracture of fifth metatarsal in zone II 


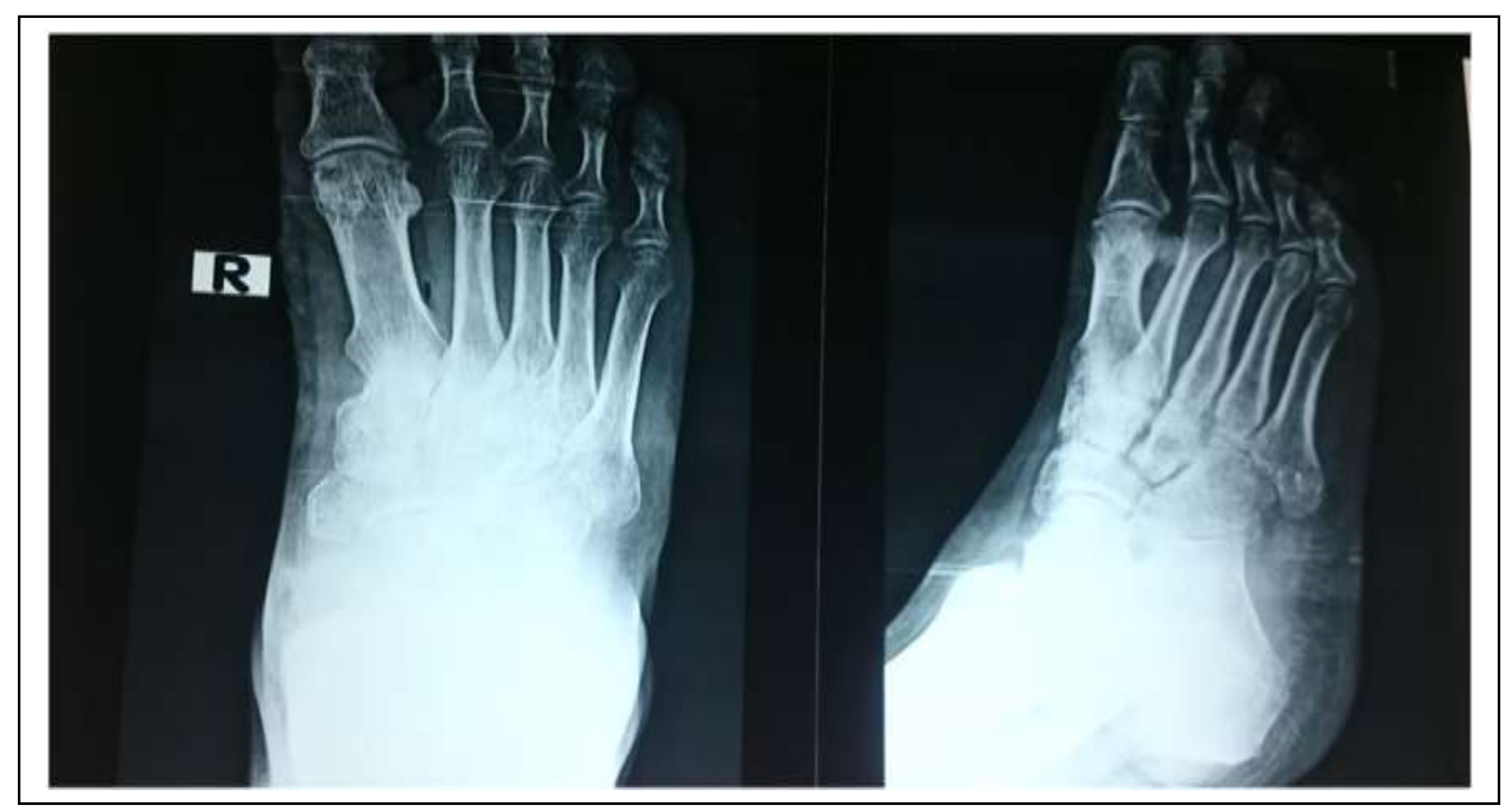

(A)

(B)

Fig (7) (a) Oblique (b) Anteroposterior of the patient after removal of cast after 10 weeks, showing radiological union.

\section{Discussion}

Fractures of the proximal fifth metatarsal, at the junction of diaphysis and metaphysis present difficulty in treatment. Sir Robert Jones originally described the fracture in 1902 when he reported 4 cases, including his own[9].

In 1927 Carp noted the difficulty in achieving union of proximal fifth metatarsal fractures [10].

A review of literature reveals considerable variability in the results obtained with non operative treatment.

The main goal of this study was to find out whether early screw fixation or conservative treatment is better and effective as a treatment modality in the case of acute Jones fracture of base of $5^{\text {th }}$ metatarsal. Early screw fixation can be strongly recommended in those who want to return to normal activity earlier. Our patient population was an active group ranging from (18-60) years in cast group and ranging from (18-60) years in operative group. Patients in both groups were productive and active.

We used radiographic classification of Dameron, Lawrence and Quill. The surgical group resulted in treatment success with average clinical union 7 weeks and the cast group had average union of 12 weeks.

The average duration of cast application in conservative group was 6 weeks, while in operative group we applied compression bandage after the surgical management. The average follow up duration was 6 months(range from 4-12 months)

The complications in the cast group are more than the operative group. Conservative group showed nonunion in five patients, Sudeck's atrophy in one patient. Operative group showed union in all patients with one patient with superficial infection treated by oral antibiotics.

T.S.Mologne [11] which is comparable with our study, studied 37 Jones fracture mean follow up 25.3 months (range, 15-42 months). Eight of 18 (44\%) in cast group were considered treatment failures: 5 nonunion, 1 delayed union, and 2 refractures. One of 19 patients in the surgery group was considered a treatment failure. For the surgery group, the median time to union and return to sports was 7.5 weeks. For the cast group, the median time to union 14.5 weeks. The Mann-Whitney test showed statistically significant difference between the groups in both parameters, with $\mathrm{P}<0.001$.

This agrees of the findings of [12] who studied 31 Jones fracture Mean follow up was 12 months (ranges 6-18 months). Six out of 16 patients $(37.5 \%)$ in the cast group were considered treatment failure ( 3 nonunion, 3 delayed union.). The 15 patients who underwent surgery were considered treatment success with some minor complications. In surgery group, the median time to clinical union and return to normal activity were 8 - 9 weeks; whereas in cast group, the median times to clinical union and return to normal activity was 14 weeks.

Andrew[13]published a systematic review of twentysix studies of which 22 were level (4) evidence, with one randomized control trial. Return to sport activity after intra-medullary screw fixation for acute fracture ranged from 4 to 18 weeks. The non-operative group had a union of $76 \%$ whereas the fracture treated operatively with intra medullary screw fixation had a union of $96 \%$. Delayed unions treated non-operatively had a union rate of $44 \%$ and $97 \%$ in treated operatively group. Non-unions treated with screw fixation healed $97 \%$ cases.

Vivek Mahajan [14]reported 23 patients healed following bicortical fixation with mean 6.3 weeks(4-10) average, there average AOFAS score was 94, they remove the implant after an average 23 weeks later, which is comparable with our results and it proves that intramedullary screw fixation is better as it needs no removal.

Marta Martina [15] reported 11 males and 6 females with type II and III Jones fractures fixed with $4.0 \mathrm{~mm}$ cannulated compression screw, had a mean healing after 
surgery $7.3,7.5$ weeks respectively and all returned to previous levels of activity no reports of delay union, nonunion or refracture, which agrees with our study.

J.H.Kavanaugh [16]reported significant complications with using non-cannulated screws such as screw breakage and a screw missing the medullary canal.

G.B.Wu [17]for example, compared the effectiveness of operative and non-operative interventions on 41 young adults, during a one year follow up. The authors reported that internal fixation of an avulsion fracture $(2$ to $3 \mathrm{~mm}$ displacement) allowed substantial enhancements in the clinical outcomes such as rate and duration of union as compared to the group administered immobilization with a plaster cast. Likewise.[11],[18], [12] also reported a higher success rate of operative interventions as compared to non-operative interventions.

J.Y.Park [19]reported that with early weight-bearing no differences in the AOFAS and VAS scores between conservative and operative treatment in patient with acute fifth metatarsal base fractures. Park et al reviewed 86 patients with acute fifth metatarsal base fractures. Conservatively treated late or early weight-bearing patients were assigned to Group A or C, respectively. Operatively treated late or early weight-bearing patients were assigned to Group B or D, respectively. Results were evaluated by clinical union, bone resorption, and the American Orthopedic Foot and Ankle Society (AOFAS) and Visual Analogue Scale (VAS) scores. All of the 4 groups had bone union at a mean of 6.9 weeks (range, 5.1-15.0). There were no differences between the groups in the AOFAS and VAS scores. In the early weight-bearing groups, there were fewer cases of bone resorption, and the bone unions periods were earlier.

Y.Wang [20]this review and meta-analysis recommend the use of operative interventions for managing the fifth metatarsal's base fracture. Which agree with our study.The study reports beneficial effects of operative interventions as compared to non-operative interventions for reducing the rate of non-union, duration of union, duration of return to activity, duration of return to sport, visual analog scale and increasing the American orthopedic foot and Ankle scale score.

The management of fracture at the base of the fifth metatarsal is a challenging avenue for an orthopedic because of its peculiar vasculature. It has been documented that the presence of the watershed-region at the base of fifth metatarsal can substantially lower the success rate concerning the rate of union especially with non-operative interventions. Due to this, the past decades have seen a substantial rise in the use of operative interventions for managing fractures at the base of the fifth metatarsal. The use of invasive interventions has primarily been favored because of their ability to provide a rigid fixation to the fractured surfaces while preserving the retrograde blood supply to the vascular insufficient areas [19].

Challenge faced in this study was lack of compliance of the patients especially in the cast group when instructed not to weight bear, patients came in the follow up with torn cast from the planter aspect denoting early weight bearing. Care of foot hygiene of the patients in the operative group to prevent infection. Limitation of study include small number of patients and short term follow up.

\section{Conclusion}

Early screw fixation is safe and effective method for treatment of acute Jones fracture of base of $5^{\text {th }}$ metatarsal. Early surgical treatment results in earlier clinical union and allows patients to return to normal daily activities more than the cast treatment which results in high rate of nonunion.

\section{References}

[1] B.A. Pterisor, Ekrol 1, Court-Brown C.The epidemiology of metatarsal fractures. Foot and Ankle Int, Vol.27, PP.172-4,2006.

[2] S.Rammelt, J.Heineck, H.Zwipp. Metatarsal fractures. Injury, Vol.35(suppl2), PP.SB77SB86,2004.

[3] E.W.Zwister, R.S.Breedervel. Fractures of the fifth metatarsal; diagnosis and treatment. Injury, Vol.41, PP.555-62, 2010.

[4] J.M.Kane, K.Sandrowski , H.Saffel. The Epidemiology of Fifth Metatarsal Fracture. Foot Ankle Spec ,2015.

[5] J.Maquirriain, J.P.Ghisi. The incidence and distribution of stress fractures in elite tennis players. Br J Sports Med, Vol.40, PP.454-459 discussion 459, 2006.

[6] R.I.Jones. Fracture of the base of the fifth metatarsal bone by indirect violence. Ann Surg, Vol.35(6), PP.697-700.2,1902.

[7] B.J.F.Dean, A.Kothari , H.Uppal. The Jones fracture classification, management, outcome, and complications: a systematic review. Foot Ankle Spec, Vol. 5, PP.256-259,2012.

[8] J.J.Cook, E.A.Cook, B.I.Rosenblum. Validation of the American College of Foot and Ankle Surgeons scoring scales. The Journal of Foot and Ankle Surgery, Vol.50(4), PP.420-429,2011.

[9] R.Jones. fractures of the base of the fifth metatarsal bone by indirect violence .Ann surg, Vol.35, PP.697-702,1902.

[10]L.Carp. fracture of fifth metatarsal bone with special reference to delayed union. Ann surg, Vol.86, PP.302-320,1927.

[11]T.S.Mologne, J.M.Lundeen, M.F.Clapper. Early screw fixation versus casting in the treatment of acute Jones fractures. Am J Sports Med, Vol.33(7), PP.970-5,2005.

[12]B.R.Adhikari, R.Thakur, G.Gurung. Comparative Study of Early Screw Fixation Versus Cast Application on the Treatment of Acute Jones Fracture. Postgraduate Medical Journal of NAMS, Vol.10, PP.2,Dec,2010.

[13]J.Andrew, Roch, D.F.James, Cladder. Treatment and return to sport following a Jones fracture of the fifth metatarsal: a systematic review. Knee surg sports trumatol arthrosc. Jun, Vol.21(6), PP.1307-15,2013. 
[14] Vivek Mahajan, Hyun Wook Ghung, Jin Soo Suh. fractures of proximal 5 th metatarsal percutaneous bicortical fixation. clin in orthopedic surgery, Vol.3, PP.140-143,2011.

[15] Marta Martina, Maanuel Alexandre, Richardo Jorge. Intramedullary screw fixation of proximal $5^{\text {th }}$ metatarsal fracture in athelets. Acta ortop bras, Vol.20(5), PP.262-265,2012.

[16] J.H.Kavanaugh, T.D.Brower, R.V.Mann. The Jones fracture revisited. J Bone Joint Surg Am, Vol.60(6), PP.776-782,1978.

[17] G.B.Wu , B.Li , Y.Yang Comparative study of surgical and conservative treatments for fifth metatarsal base avulsion fractures in young adults or athletesJournal of Orthopaedic Surgery , Vol.26(1), PP.1-5,2017.
[18] S.M. Sokkar, A.Abdelkafy. Operative versus nonoperative treatment of fifth metatarsal fracture in nonprofessional athletes. Egypt Orthop J, Vol.51, PP.319-22,2016.

[19]J.Y.Park , Hyong-Nyun Kim, Yoon-Suk Hyun. Effect of Weight-Bearing in Conservative and Operative Management of Fractures of the Base of the Fifth Metatarsal Bone Hindawi BioMed Research International, Article ID, Vol. 1397252, PP.6,2017.

[20] Y.Wang, X.Gan, K.Li. Comparison of operative and non-operative management of fifth metatarsal base fracture: A meta-analysis. PLoS ONE , Vol.15(8), PP. e0237151,2020. 